\title{
Mechanical Ventilation in Pregnancy Due to COVID-19: A Cohort of Three Cases
}

\author{
Elizabeth Lucarelli, MD ${ }^{1}$ Claudia Behn, MD ${ }^{2}$ Susan Lashley, MD ${ }^{1}$ Dorothy Smok, MD ${ }^{1}$ \\ Carlos Benito, MD, MPH ${ }^{2}$ Yinka Oyelese, MD $^{1}$
}

${ }^{1}$ Atlantic Health System, Morristown, New Jersey

${ }^{2}$ St Peter's University Hospital, New Brunswick, New Jersey

Am J Perinatol 2020;37:1066-1069.
Address for correspondence Yinka Oyelese, MD, Atlantic Maternal Fetal Medicine, 435 South Street Suite 380, Morristown, NJ 07960 (e-mail: yinkamd@aol.com).
Abstract
Keywords
- COVID-19
- coronavirus
- pregnancy complications
- mechanical ventilation
- pregnancy
- intubation

\begin{abstract}
We describe our experience with three pregnant women with novel coronavirus disease 2019 (COVID-19) who required mechanical ventilation. Recent data suggest a mortality of $88 \%$ in nonpregnant patients with COVID-19 who require intubation and mechanical ventilation. The three women we report were intubated and mechanically ventilated during pregnancy due to respiratory failure and pneumonia resulting from COVID-19. After several days of ventilation, all three were successfully weaned off mechanical ventilation and extubated, and are continuing their pregnancies with no demonstrable adverse effects. Our experience suggests that the mortality in pregnant women with COVID-19 requiring mechanical ventilation is not necessarily as high as in nonpregnant patients with COVID-19.
\end{abstract}

\section{Key Points}

- Coronavirus disease 2019 (COVID-19) is now a pandemic.

- COVID-19 may cause pneumonia or respiratory failure in pregnant women.

- Approximately 5\% of women with COVID-19 will develop severe or critical disease.

- Mechanical ventilation in pregnant women may not necessarily result in high mortality rates.

Novel coronavirus disease 2019 (COVID-19) has now become a pandemic, with over 1.5 million cases and nearly 100,000 deaths reported in the United States to date and invariably has come to affect pregnant women. ${ }^{1}$ New Jersey is second only to New York in the number of cases and deaths resulting from COVID-19. As such, at our centers, we have had significant experience with a large number of patients with COVID-19. Morristown Medical Center and St. Peter's University Medical Center are tertiary perinatal centers who care for a large population of pregnant women in central New Jersey.

Until recently, little has been known about outcomes in pregnancies affected by COVID-19. ${ }^{2}$ Approximately 5 to $10 \%$ of patients with COVID-19 will require intubation and me- chanical ventilation. ${ }^{2}$ In the general population, mortality in patients requiring mechanical ventilation has been reported as approaching $90 \%{ }^{3}$ We report on three women with COVID-19 who required mechanical ventilation while pregnant. These were the only pregnant women with COVID-19 who required mechanical ventilation at our institutions between March 31, 2020 and May 20, 2020.

\section{Case 1}

A 38-year-old G3P1011 woman presented to the emergency department (ED) at 28 weeks of gestation with complaints of persistent dry cough, pleuritic chest pain, and shortness of breath for 5 days, and diarrhea for 2 days. She reported that received

May 25, 2020

accepted after revision

May 28, 2020

published online

June 16, 2020
Copyright $\odot 2020$ by Thieme Medical

Publishers, Inc., 333 Seventh Avenue, New York, NY 10001, USA. Tel: +1(212) 760-0888.
DOI https://doi.org/ 10.1055/s-0040-1713664. ISSN 0735-1631. 
both her parents who she lived with had similar respiratory symptoms. She denied any fevers or chills.

At the time of presentation, she was afebrile. Her peripheral oxygen $\left(\mathrm{SpO}_{2}\right)$ saturation was $96 \%$ on room air. Her electrocardiogram showed sinus tachycardia. The fetal heart rate was 160 beats per minute. Her white cell count was $11,000 / \mu \mathrm{L}$; lymphocytes were $4.7 \%$ (normal $15-40 \%$ ); hemoglobin was $11 \mathrm{~g} / \mathrm{dL}$; and her platelet count was $210,000 / \mu \mathrm{L}$. Her pharyngeal screens for influenza and streptococcus $A$ were negative. A chest X-ray showed patchy infiltrates in the left mid and lower lung and right medial lung base. A computed tomography (CT) angiogram of her chest showed dense consolidations and multifocal ground-glass opacities in the lower lobes and inferior upper lobes bilaterally. A COVID-19 real-time polymerase chain reaction (RT-PCR) nasopharyngeal swab was performed. The patient was admitted to the medicine service with presumed COVID-19 disease. She was started on intravenous ceftriaxone $500 \mathrm{mg}$ IV and azithromycin $250 \mathrm{mg}$ p.o. daily, and maternal fetal medicine was consulted.

Following admission, the patient's $\mathrm{SpO}_{2}$ dropped as low as $82 \%$ while she was coughing, and her respiratory rate was $30 /$ minute at rest, butrose as high as 50 to 65/minute with coughing. Her $\mathrm{SpO}_{2}$ improved to 89 to $96 \%$ on $6 \mathrm{~L}$ of $\mathrm{O}_{2}$ by nasal cannula. On the second day of admission, the patient was placed on a $50 \%$ Ventimask and her $\mathrm{SpO}_{2}$ was 95 to $99 \%$, except when she was coughing, when it decreased to approximately $80 \%$. The infectious disease service was consulted.

The patient's COVID-19 test result came back positive. She began to complain of decreased fetal movement. A fetal biophysical profile was $8 / 8$. The fetus was appropriately grown with normal amniotic fluid volume. The patient was treated with hydroxychloroquine p.o. $400 \mathrm{mg}$ b.i.d. for two doses and then $200 \mathrm{mg}$ b.i.d. for eight doses.

However, she continued to have desaturations of her $\mathrm{O}_{2}$, especially with coughing, and she was upgraded to a nonrebreather mask at $100 \%$ oxygen. She then developed a fever of $38.2^{\circ} \mathrm{C}$. The critical care team was consulted. Methylprednisolone (125 mg IV for one dose which followed by $40 \mathrm{mg}$ IV b.i.d. and tapered over 5 days to $20 \mathrm{mg}$ IV daily for one dose) was started for maternal treatment of worsening hypoxemia and tachypnea, and betamethasone (12 $\mathrm{mg}$ IM single dose repeated for one dose 24 hours later) was given to induce fetal lung maturation. The patient was transferred to the medical ICU, at which time her $\mathrm{paO}_{2}$ was $83 \mathrm{~mm} \mathrm{Hg}$, her $\mathrm{paCO}_{2}$ was $28 \mathrm{~mm} \mathrm{Hg}$, her $\mathrm{pH}$ was 7.37, her $\mathrm{HCO}_{3}$ was $15.9 \mathrm{mmol} / \mathrm{L}$, and her $\mathrm{SpO}_{2}$ was $96 \%$ on a nonrebreather mask.

Because of severe respiratory distress, tachypnea of 53 respirations per minute, and persistent hypoxia with $\mathrm{SpO}_{2}$ in the 80 s on a $100 \%$ nonrebreather mask, a decision was made to intubate her and start her on mechanical ventilation. The $\mathrm{pH}$ goal was set at greater than 7.3 and $\mathrm{FiO}_{2}$ was titrated to maintain $\mathrm{SpO}_{2}$ over $95 \%$. An application was made to use Remdesivir for compassionate use, which was approved, and the patient was started on Remdesivir (200 mg IV every 12 hours $\times$ two doses and then $100 \mathrm{mg}$ IV daily $\times$ nine doses). The patient remained intubated and ventilated mechanically for 5 days.
Tube feeds were started. $\mathrm{SpO}_{2}$ remained at $100 \%$ while on mechanical ventilation. A bedside ultrasound revealed a breech presenting fetus with a fetal heart rate of 170 beats/minute, with normal amniotic fluid. The patient did well, and because of decreasing oxygen demands, she was extubated after 5 days of mechanical ventilation. Following extubation, her $\mathrm{SpO}_{2}$ was 93 to $99 \%$ on a Ventimask. Her azithromycin, ceftriaxone, and hydroxychloroquine courses were completed. She was transferred from the ICU to a COVID-19 unit, and her methylprednisolone was tapered. The ceftriaxone was discontinued after 7 days. Her oxygen requirements continued to decrease, and by hospital day 10 , she was back on room air. The patient completed her course of Remdisivir on hospital day 12. Fetal ultrasound showed a biophysical profile of $8 / 8$. The patient was discharged home on hospital day 14 in stable condition, at 30 weeks of gestation. She is currently at 34 weeks of gestation and is doing well, with no complaints.

\section{Case 2}

A 26-year-old G2P1001 woman presented to the ED at 29 weeks and 6 days of gestation with shortness of breath, cough, generalized body aches, abdominal pain, fever, and nausea. The patient was tachycardic, with a heart rate of 130 beats per minute. There was also fetal tachycardia in the 190 s, which eventually decreased to the 150 s. Her chest Xray was normal and her rapid influenza test was negative. A ventilation-perfusion scan showed a low risk for pulmonary embolism. She felt better and was discharged home with instructions to self-quarantine. She represented 2 days later at a gestational age of 30 weeks and 1 day with generalized muscle and body aches, shortness of breath, nausea, vomiting, and fever. A repeat chest X-ray showed superimposed peribronchial thickening and multifocal pneumonia. A nasopharyngeal swab was taken for a RT-PCR COVID-19 test, and she was treated as a person under investigation. She was treated with a single dose of intravenous ceftriaxone $1 \mathrm{~g}$ IV and azithromycin $500 \mathrm{mg}$ p.o. daily for 2 days which followed by $250 \mathrm{mg}$ p.o. daily for 4 days) for presumptive multifocal community acquired pneumonia. She was afebrile and her $\mathrm{SpO}_{2}$ was $97 \%$ on room air; therefore, she was discharged home to self-quarantine. The following day she returned to the ED with severe dyspnea at rest and profound fatigue. She was readmitted to the hospital. At this time, she had a fever as well as tachycardia and tachypnea. She was started on high flow nasal oxygen to maintain her $\mathrm{SpO}_{2}$ at greater than $>92 \%$. She also received Heparin 5000 units SQ every 12 hours for thromboprophylaxis. She was given intravenous fluids, tylenol, intravenous ceftriaxone $1 \mathrm{~g}$ IV daily, and continued her azithromycin (500 mg daily for 2 days which followed by $250 \mathrm{mg}$ p.o. daily for 4 days). Her COVID-19 test returned positive, and hydroxychloroquine (400 b.i.d. for 1 day which followed by $200 \mathrm{mg}$ p.o. b.i.d. for 4 days) was added to her therapy. Total 3 days later, her repeat chest X-ray showed more dense hazy airspace opacities in the bilateral mid and lower lungs, and she was given furosemide $20 \mathrm{mg}$ IV. She was then started on high-flow nasal cannula with $45 \% \mathrm{O}_{2}$, on 
which she maintained an $\mathrm{SpO}_{2}$ of 95 to $98 \%$. She continued to worsen and because of acute respiratory failure, she was intubated in the intensive care unit (ICU). She remained intubated for 3 days. She required multiple regimens of sedatives to help her tolerate intubation and to decrease her respiratory distress. She was treated for 5 days with azithromycin, hydroxychloroquine, and ceftriaxone. She was also treated with beclomethasone $12 \mathrm{mg}$ every 24 hours for two doses, then prednisone 40 b.i.d., and transitioned to methylprednisolone $40 \mathrm{mg}$ b.i.d. During her intubation, she received tube feeds and had a Foley catheter placed. Because of clinical improvement and lessening oxygen demands, she was extubated after 3 days on the ventilator and transitioned to oxygen by high-flow nasal cannula. The following day, she was transitioned to $3 \mathrm{~L} \mathrm{O}_{2}$ by nasal cannula and downgraded from the ICU to the antepartum COVID unit. While she had increases in hypertransaminases, her blood pressure remained normal and there was no other evidence of preeclampsia or hemolysis, elevated liver enzymes, and low platelet count syndrome. At 31 weeks and 4 days, her $\mathrm{SpO}_{2}$ was 97 to $99 \%$ on room air, and her respiratory rate was $20 /$ minute. She was discharged home with a prednisone taper. She was given strict quarantine instructions. Nonstress tests throughout admission were reactive and reassuring. She is doing well at 35 weeks of gestation.

\section{Case 3}

A 46-year-old female G6P2032 with a pregnancy resulting from in vitro fertilization with a donor egg initially presented at 23 weeks and 5 days of gestation with cough and shortness of breath, and was tested for COVID-19 (nasopharyngeal swab with RT-PCR). Her influenza test was negative. Because she had normal $\mathrm{SpO}_{2}$, she was treated with azithromycin $500 \mathrm{mg}$ p.o. followed by $250 \mathrm{mg}$ p.o. daily for 5 days and augmentin ( $875 \mathrm{mg}$ p.o. b.i.d. for 5 days) and managed as an outpatient. At home, her dyspnea worsened. In the interim, her COVID-19 test returned positive. She represented to the hospital with worsening shortness of breath and decreased $\mathrm{SpO}_{2}$ of 84 to $85 \%$ at home. On admission, she was diagnosed with acute hypoxic respiratory failure and had an $\mathrm{SpO}_{2}$ of $85 \%$ on room air. A chest CT angiogram was negative for pulmonary embolism but showed multifocal areas of bilateral peripheral ground glass opacities with bilateral dependent consolidations and cardiomegaly. She was placed on a high-flow nasal cannula at $60 \%$, on which her $\mathrm{SpO}_{2}$ improved to 92 to $93 \%$. She was started on hydroxychloroquine $400 \mathrm{mg}$ p.o. b.i.d., followed by $200 \mathrm{mg}$ p.o. b.i.d. $\times 6$ days. She had already received an outpatient course of azithromycin and augmentin and was given a single dose of ceftriaxone $1 \mathrm{~g}$ IV while in the ED. On hospital day 2 , she was intubated due to persistent $\mathrm{O}_{2}$ desaturation to the 80 s despite receiving supplemental oxygen by high-flow nasal cannula. A central venous line was placed, as well as a feeding tube. She received was treated with azithromycin, methylprednisolone $40 \mathrm{mg}$ IV b.i.d. and a single intravenous $800 \mathrm{mg}$ dose of tocilizumab (anIL6 antagonist) in addition to the hydroxychloroquine. She was also treated with Lovenox $40 \mathrm{mg}$ daily for thromboprophylaxis. She received betamethasone (12 mg IM, 2 doses 24 hours apart) to promote fetal lung maturation. Daily fetal heart rate monitoring was performed. The following day, she was noted to have oliguria, an elevated creatinine of $4.01 \mathrm{mg} / \mathrm{dL}$, and a potassium level of 5.7 $\mathrm{mmol} / \mathrm{L}$, and was diagnosed with acute kidney injury, thought to be secondary to COVID-19, with superimposed contrast injury. Continuous dialysis was commenced after a central venous line catheter was placed for continuous venovenous hemodialysis (CVVHD). However, despite the thromboprophylaxis, the blood repeatedly coagulated in the dialysis machine. Thus, the patient was started on a continuous heparin drip, following which CVVHD was successfully started. Because of anemia, the patient received one unit of packed red blood cells. Her aspartate and alanine aminotransferase levels became elevated, and there was a suspicion of multiorgan dysfunction. Because of decreasing oxygen demands, and patient improvement, after 8 days of ventilation, the patient was successfully extubated and started on a high-flow nasal cannula $8 \mathrm{~L}$ with $100 \% \mathrm{SpO}_{2}$. For the next 4 days, she received intermittent dialysis. Five days after extubation, she was maintaining an $\mathrm{SpO}_{2}$ of $96 \%$ on room air. In addition, her creatinine was improving. She was then placed on intermittent hemodialysis. She later left the institution at 26 weeks of gestation. She had a follow-up as an outpatient at 28 weeks and 4 days of gestation at which time she had no complaints, and her liver function tests and creatinine had returned to normal without dialysis.

\section{Discussion}

Until recently, there has been little data to guide those providing care to pregnant women with COVID-19. ${ }^{4}$ It was initially thought, based on high rates of severe morbidity and death among pregnant women who develop influenza, that pregnant woman affected with COVID-19 may suffer greatly increased morbidity and mortality. ${ }^{1}$ Recent data suggest that pregnant women do not have worse outcomes than the general population. ${ }^{2}$ However, a large cohort of 5,700 patients with COVID-19 from a New York hospital group found a mortality of $88.1 \%$ among nonpregnant COVID-19 patients who were intubated and received mechanical ventilation. ${ }^{3}$ Because pregnant women with severe disease may require intubation and mechanical ventilation, this obviously raises concern as to their outcomes. ${ }^{4}$ Indications for intubation in pregnant women include acute respiratory distress syndrome (ARDS) and respiratory failure. ${ }^{4-6}$

In our three cases, the mothers did well despite intubation and requirement for mechanical ventilation. Gestational ages at intubation were between 23 and 29 weeks, and duration of mechanical ventilation was 3 to 8 days. Furthermore, while their pregnancies are ongoing, there has been no evidence of fetal distress or of any adverse effects on their fetuses. While only very limited data are available on pregnant women with COVID-19 who have been intubated and ventilated, our three cases suggest that COVID-19 in pregnancy requiring ventilation is not necessarily associated with mortality. The reasons for the discrepancy with the prior 
studies finding poor outcomes in the overall population of COVID-19 patients who are ventilated may have to do with the fact that pregnant women are generally younger. COVID19 has much higher case-fatality rates in the older population. The median age of mortality in the New York cohort was 68 years; in that study, mortality rates for patients who received mechanical ventilation were 76.4 and $97.2 \%$ in the 18 to 65 and older than 65 age groups, respectively. ${ }^{3}$

Furthermore, while COVID may affect both genders, the disease appears to have worse outcomes in men. In the New York cohort, two-thirds of patients requiring hospitalization were male. ${ }^{3}$ None of our three patients required delivery. A prior study found that $75 \%$ of women with critical COVID-19 infection in pregnancy were delivered iatrogenically. ${ }^{4} \mathrm{Fi}-$ nally, in all three women, the indications for mechanical ventilation were respiratory failure and pneumonia. None of them had ARDS. It is likely that pregnant women who develop ARDS and require mechanical ventilation may have worse outcomes. Our findings suggest that women with COVID-19 who require mechanical ventilation do not necessarily need to be delivered. In addition, our limited experience showing favorable outcomes in these women may help guide practitioners caring for pregnant women with COVID-19 who may have to be treated with mechanical ventilation.

\section{Conflict of Interest}

None declared.

\section{References}

1 Rasmussen SA, Smulian JC, Lednicky JA, Wen TS, Jamieson DJ. Coronavirus Disease 2019 (COVID-19) and pregnancy: what obstetricians need to know. Am J Obstet Gynecol 2020;222(05):415-426

2 Breslin N, Baptiste C, Gyamfi-Bannerman C, et al. COVID-19 infection among asymptomatic and symptomatic pregnant women: two weeks of confirmed presentations to an affiliated pair of New York City hospitals. Am J ObstetGynecol MFM 2020:100118. Doi: $10.1016 /$ j.ajogmf.2020.100118

3 Richardson S, Hirsch JS, Narasimhan M, et al; and the Northwell COVID-19 Research Consortium. Presenting characteristics, comorbidities, and outcomes among 5700 patients hospitalized with COVID-19 in the New York City area. JAMA 2020:e206775. Doi: 10.1001/jama.2020.6775

4 Pierce-Williams RAM, Burd J, Felder L, et al. Clinical course of severe and critical COVID-19 in hospitalized pregnancies: a US cohort study. Am J Obstet Gynecol MFM 2020:100134. Doi: 10.1016/j.ajogmf.2020.100134

5 Kasraeian M, Zare M, Vafaei H, et al. COVID-19 pneumonia and pregnancy; a systematic review and meta-analysis. J Matern Fetal Neonatal Med 2020;19:1-8

6 Schnettler WT, Al Ahwel Y, Suhag A. Severe ARDS in COVID-19infected pregnancy: obstetric and intensive care considerations. Am J Obstet Gynecol MFM 2020:100120. Doi: 10.1016/j. ajogmf.2020.100120 